\title{
Efficacy of Transarterial Chemoembolisation with or without Antiviral Therapy for Patients with Hepatocellular Carcinoma after Radical Hepatectomy
}

\author{
Yin Zhu, ${ }^{1}$ Pei-Jing Cui, ${ }^{2}$ Jing Yao, ${ }^{1}$ Zheng-Yun Zhang, ${ }^{1}$ and Jun Yang $\mathbb{D}^{1}$ \\ ${ }^{1}$ Department of Surgery, Affiliated Sixth People's Hospital, Shanghai Jiao Tong University, Shanghai 200233, China \\ ${ }^{2}$ Department of Geriatrics, Ruijin Hospital, School of Medicine, Shanghai Jiao Tong University, Shanghai 200025, China \\ Correspondence should be addressed to Jun Yang; yangjuns@foxmail.com
}

Received 18 October 2017; Revised 4 January 2018; Accepted 9 January 2018; Published 1 April 2018

Academic Editor: Nicola Silvestris

Copyright ( 2018 Yin Zhu et al. This is an open access article distributed under the Creative Commons Attribution License, which permits unrestricted use, distribution, and reproduction in any medium, provided the original work is properly cited.

\begin{abstract}
Objective. This study aimed at assessing the effects of transcatheter arterial chemoembolisation (TACE) and antiviral therapy on improving the prognosis of patients with hepatocellular carcinoma (HCC) after radical hepatectomy. Methods. This study reviewed the data of 120 patients with HCC who received either radical hepatectomy alone (control group), radical hepatectomy with postoperative TACE (TACE group) or radical hepatectomy with combined postoperative TACE, and antiviral therapy (combined group) from January 2000 to May 2015. To reduce the impact of the possible biases on the conclusion of this study to the minimum, the cases with similar demographic and clinicopathological characteristics were collected and 40 cases were assigned into each group. Recurrence, disease-free survival (DFS), and overall survival (OS) rates were compared. Results. Median follow-up period was 54.26 \pm 22.65 months with a range of 17-110 months. Recurrence after radical surgery was observed for 39 (97.5\%) patients in the TACE group, 32 (80\%) in the combined group, and 40 (100\%) in the control group with median recurrence duration of 33, 43, and 16.5 months, respectively. Postoperative TACE with or without antiviral therapy significantly prolonged the DFS rate compared with radical hepatectomy alone $(P=0.000)$. TACE combined with antiviral therapy significantly extended the DFS rate compared with TACE alone $(P=0.008)$. Postoperative TACE with or without antiviral therapy also significantly prolonged the OS rate compared with radical hepatectomy alone $(P=0.000)$. In addition, antiviral therapy combined with TACE significantly extended the 5-year OS rate of patients compared with individual TACE and radical hepatectomy $(67.5 \%$ versus $55 \%$ and $2.5 \% ; P=0.032)$. Conclusion. TACE is an appropriate therapy for HCC patients after radical hepatectomy. When combined with antiviral therapy, this treatment may further prolong the recurrence time and thus lead to high DFS and OS rates.
\end{abstract}

\section{Introduction}

Hepatocellular carcinoma (HCC) is one of the most common malignancies worldwide. This disease accounts for $5.6 \%$ of all human cancers, and its incidence is gradually increasing [1]. In China, HCC mortality ranked second to stomach cancer. The main therapies for HCC are surgical interventions (tumour resection and liver transplantation), percutaneous interventions (ethanol injection and radiofrequency thermal ablation), transarterial interventions (embolisation, chemoperfusion, or chemoembolisation), and therapeutic drugs, including gene and immune therapy [2]. Among these treatments, surgical resection is the first-line therapeutic option for patients with HCC. Unfortunately, the long-term survival, even after curative liver resection, is unsatisfactory because of the high incidence of postoperative, specifically intrahepatic, tumour recurrence [3]. The 3- and 5-year recurrence rates are $62.3 \%-93.2 \%$ and $79.0 \%$, respectively [4]. Several approaches were developed to decrease or delay the incidence of recurrence and the effects of HCC. As first reported by Goldstein, transcatheter arterial chemoembolisation (TACE) directly kills the tumour cells and blocks the blood supply to the tumour [5]. This treatment is now widely used in Chinese clinics to prevent the recurrence of HCC 
after surgery. However, its effects are still controversial and difficult to determine. Meta-analysis suggested that postoperative adjuvant TACE seems promising for participants with $\mathrm{HCC}$ with risk factors (multiple nodules of $>5 \mathrm{~cm}$ or vascular invasion) [6]; however, other studies indicated that TACE can damage the remnant liver, harm the liver function, and interfere with immune function, which may increase the risk of reactivating hepatitis $\mathrm{B}$ virus (HBV) replication; all these effects can worsen the prognosis [7-9]. A study conducted in South Korea showed that TACE reactivates HBV replication in HCC patients that are HBsAg-positive. Higher HBV viral load may trigger HCC recurrence after liver resection [8]. Hung et al. [10] also found that a HBV viral load greater than $2000 \mathrm{IU} / \mathrm{ml}$ is associated with an odds ratio as high as 22.3 for HCC recurrence after liver resection. Antiviral therapy using nucleoside analogues is recently found to effectively supress HBV replication and reduce high viral load [11]. A small nonrandomised study from Japan found that lamivudine therapy is beneficial to patients that were initially treated with partial hepatectomy or radiofrequency ablation for HBV-related HCC [12]. Other studies showed that the combination of postoperative TACE and antiviral therapy may be more effective than TACE alone in improving the prognosis and reducing the recurrence in patients with HBV-related HCC after hepatectomy $[13,14]$.

Despite the increasing use of postoperative TACE and antiviral therapy for patients with HCC, a universally accepted guideline for the application in patients treated with radical hepatectomy is not yet established. Therefore, a retrospective review of the medical records of patients who received either radical hepatectomy alone, radical hepatectomy with postoperative TACE or radical hepatectomy with combined postoperative TACE, and antiviral therapy was conducted. The effects of TACE and antiviral therapy on improving the prognosis of patients with HCC after radical hepatectomy were assessed by comparing the recurrence, disease-free survival (DFS), and overall survival (OS) rates.

\section{Methods}

2.1. Patients. A total of 120 patients with chronic $\mathrm{HBV}$ infection were diagnosed with initial HCC in our department between January 2000 and May 2015. A retrospective analysis was conducted using the medical records of patients who satisfied the following inclusion criteria: (1) radical hepatectomy with histopathologically confirmed safety surgical margin of $\geq 2 \mathrm{~cm}$; (2) histopathological examination confirming no major vascular or bile duct invasion or tumour thrombus in portal vein; (3) preoperative imaging diagnosis and surgical finding verifying no distant extrahepatic metastases; (4) chronic hepatitis B infection with $\mathrm{HBV}$ DNA level $>10^{3}$ copies $/ \mathrm{ml}$ and without other viral hepatitis infections; (5) no evidence indicative of cancerisation from drug, alcoholic, fatty liver, or autoimmune hepatitis; and (6) without any other organ dysfunction or failure. Those patients who underwent preoperative treatments, such as TACE, antiviral therapy, or systemic chemotherapy, were excluded. Informed consent from all patients was obtained before performing any treatment.
2.2. Treatment. Hepatectomy was conducted by only one surgical team. Each patient received radical hepatectomy according to the anatomical principles of resection whenever possible. Intraoperative ultrasonography was used to identify any occult tumours that were not detected preoperatively and to confirm the relationship between the tumour and vasculobiliary structures. Parenchymal dissection was performed using ultrasonic dissectors.

2.3. Adjuvant TACE. TACE was conducted 2 months after radical hepatectomy by using the techniques described previously by Li et al. [15]. The therapeutic regimen included 5-fluorouracil (5-FU) $500 \mathrm{mg} / \mathrm{m}^{2}$, cisplatin (DDP) $40 \mathrm{mg} / \mathrm{m}^{2}$, and epirubicin (EPI) $40 \mathrm{mg} / \mathrm{m}^{2}$ with lipiodol $3 \mathrm{ml} / \mathrm{m}^{2}$ through chemoembolisation following hepatic arterial angiography. The puncture sites were compressed tightly, and the side limbs were restricted within a range of motion for at least $12 \mathrm{~h}$. Supine position was assumed for $24 \mathrm{~h}$ afterwards to prevent bleeding or hematoma. The treatment was regularly repeated after 2 months depending on the patient's response to the first one.

2.4. Antiviral Therapy. This group includes patients who fulfilled the inclusion criteria and the following had ALT level two times higher than the upper limits and with or without HBV DNA greater than $10^{4}$ copies/ml, or with HBV DNA greater than $10^{4}$ copies $/ \mathrm{ml}$. These patients received lamivudine at a dose of $100 \mathrm{mg}$ per day or entecavir $0.5-1.0 \mathrm{mg}$ per day (if resistant to lamivudine) starting from 2 weeks after radical hepatectomy.

2.5. Follow-Up. The first endpoint was DFS, starting from the surgery date until the recurrence of HCC. The second one is OS, which is defined as the date of patient's death or last census date (1 May 2015). Patients were followed up to monitor HCC recurrence. Blood tests, including liver functions (ALT, AST, ALP, TB, and TSP), $\alpha$-fetoprotein (AFP) level, and HBV DNA level, and ultrasonography were performed 1 month after surgery and then every 2 months afterwards for the first year. Intervals would later increase depending on the patient's situation. Further imaging studies such as CT or MRI were conducted if evidence of recurrence was observed. The diagnosis of recurrence or metastasis was confirmed under pathological examinations if necessary.

2.6. Statistical Analysis. SPSS 23.0 (SPSS Inc., Chicago, IL, USA) was used for statistical analysis. Demographic and clinical data were described using medians or frequencies. One-way ANOVA or chi-square test was used for the comparison of different groups. Fisher's exact test would be applied when homogeneity of variance assumption was satisfied. If the assumption failed, Brown-forsythe would be used. If $P<0.05$ when comparing the demographic and clinical data, multiple comparisons like LSD test were used to distinguish the different one among the three groups. Regression analysis was used to determine the influencing factors. Survival curves were estimated using the Kaplan-Meier method and were compared through log-rank test. All statistical tests reached statistical significance at $P<0.05$. 
TABle 1: Preoperative demographic characteristics of patients in the three groups.

\begin{tabular}{|c|c|c|c|c|c|}
\hline \multicolumn{2}{|c|}{ Preoperative patient demographic } & \multirow{2}{*}{$\begin{array}{c}\text { Control group } \\
31(77.5 \%)\end{array}$} & \multirow{2}{*}{$\frac{\text { TACE group }}{26(65 \%)}$} & \multirow{2}{*}{$\frac{\text { Combined group }}{32(80.0 \%)}$} & \multirow{2}{*}{$P$ value } \\
\hline \multirow{2}{*}{ Gender } & Male & & & & \\
\hline & Female & $9(22.5 \%)$ & $14(35 \%)$ & $8(20.0 \%)$ & \\
\hline Age $(y)$ & & $52.25 \pm 8.42$ & $51.58 \pm 8.26$ & $49.78 \pm 9.51$ & 0.427 \\
\hline \multirow{2}{*}{ Ascites } & Medically controlled & $19(47.5 \%)$ & $5(12.5 \%)$ & $0(0.0 \%)$ & \multirow{2}{*}{0.000} \\
\hline & No & $21(52.5 \%)$ & $35(87.5 \%)$ & $40(100.0 \%)$ & \\
\hline \multirow{2}{*}{ Hepatic encephalopathy } & Yes & $0(0.0 \%)$ & $0(0.0 \%)$ & $0(0.0 \%)$ & \multirow{2}{*}{1.000} \\
\hline & No & $40(100.0 \%)$ & $40(100.0 \%)$ & $40(100.0 \%)$ & \\
\hline INR & & $1.06 \pm 0.81$ & $1.05 \pm 0.75$ & $1.06 \pm 0.78$ & 0.947 \\
\hline Total bilirubin $(\mu \mathrm{mol} / \mathrm{L})$ & & $18.88 \pm 4.29$ & $19.53 \pm 3.74$ & $18.96 \pm 4.14$ & 0.739 \\
\hline Total serum protein $(\mathrm{g} / \mathrm{L})$ & & $35.93 \pm 3.72$ & $34.30 \pm 1.96$ & $35.80 \pm 2.97$ & 0.028 \\
\hline \multirow{2}{*}{ Child-Pugh class } & A & $33(82.5 \%)$ & $35(87.5 \%)$ & $36(90.0 \%)$ & \multirow{2}{*}{0.606} \\
\hline & B & $7(17.5 \%)$ & $5(12.5 \%)$ & $4(10.0 \%)$ & \\
\hline ALT (U/L) & & $44.68 \pm 9.96$ & $50.00 \pm 9.83$ & $47.95 \pm 12.48$ & 0.090 \\
\hline $\operatorname{ALP}(\mathrm{U} / \mathrm{L})$ & & $151.98 \pm 70.39$ & $149.10 \pm 91.02$ & $127.40 \pm 70.52$ & 0.308 \\
\hline $\operatorname{AST}(\mathrm{U} / \mathrm{L})$ & & $40.70 \pm 8.41$ & $44.58 \pm 10.11$ & $43.08 \pm 11.07$ & 0.217 \\
\hline $\mathrm{Cr}(\mu \mathrm{mol} / \mathrm{L})$ & & $62.48 \pm 13.06$ & $60.93 \pm 11.87$ & $62.38 \pm 12.32$ & 0.823 \\
\hline $\mathrm{WBC}\left(\times 10^{9} / \mathrm{L}\right)$ & & $4.69 \pm 0.57$ & $4.88 \pm 0.80$ & $4.93 \pm 7.95$ & 0.304 \\
\hline $\mathrm{RBC}\left(\times 10^{12} / \mathrm{L}\right)$ & & $3.47 \pm 0.36$ & $3.32 \pm 0.27$ & $3.34 \pm 0.33$ & 0.100 \\
\hline $\operatorname{PLT}\left(\times 10^{9} / \mathrm{L}\right)$ & & $88.58 \pm 19.61$ & $90.48 \pm 18.63$ & $87.73 \pm 23.37$ & 0.830 \\
\hline $\mathrm{Hb}(\mathrm{g} / \mathrm{L})$ & & $118.30 \pm 18.89$ & $113.48 \pm 14.56$ & $116.05 \pm 14.16$ & 0.406 \\
\hline $\operatorname{AFP}(n g / m L)$ & & $474.10 \pm 185.44$ & $949.80 \pm 1160.62$ & $710.76 \pm 906.18$ & 0.052 \\
\hline CEA (ng/mL) & & $2.15 \pm 0.48$ & $2.09 \pm 0.52$ & $2.24 \pm 0.77$ & 0.534 \\
\hline CA199 (ng/mL) & & $22.35 \pm 4.43$ & $20.80 \pm 4.38$ & $19.96 \pm 4.66$ & 0.058 \\
\hline \multirow{2}{*}{ HbsAg } & Positive & $40(100.0 \%)$ & $40(100.0 \%)$ & $40(100.0 \%)$ & \multirow{2}{*}{1.000} \\
\hline & Negative & $0(0.0 \%)$ & $0(0.0 \%)$ & $0(0.0 \%)$ & \\
\hline \multirow{2}{*}{$\mathrm{HbsAb}$} & Positive & $7(17.5 \%)$ & $17(42.5 \%)$ & $16(40.0 \%)$ & \multirow{2}{*}{0.034} \\
\hline & Negative & $33(82.5 \%)$ & $23(57.5 \%)$ & $24(60.0 \%)$ & \\
\hline \multirow{2}{*}{$\mathrm{HbcAb}$} & Positive & $40(100.0 \%)$ & $40(100.0 \%)$ & $40(100.0 \%)$ & \multirow{2}{*}{1.000} \\
\hline & Negative & $0(0.0 \%)$ & $0(0.0 \%)$ & $0(0.0 \%)$ & \\
\hline \multirow{2}{*}{ HbeAg } & Positive & $40(100.0 \%)$ & $40(100.0 \%)$ & $40(100.0 \%)$ & \multirow{2}{*}{1.000} \\
\hline & Negative & $0(0.0 \%)$ & $0(0.0 \%)$ & $0(0.0 \%)$ & \\
\hline \multirow{2}{*}{$\mathrm{HbeAb}$} & Positive & $40(100.0 \%)$ & $0(0.0 \%)$ & $0(0.0 \%)$ & \multirow{2}{*}{1.000} \\
\hline & Negative & $0(0.0 \%)$ & $40(100.0 \%)$ & $40(100.0 \%)$ & \\
\hline HBA DNA level & & $2.93 \times 10^{5}$ & $1.16 \times 10^{6}$ & $5.66 \times 10^{5}$ & 0.501 \\
\hline
\end{tabular}

\section{Results}

3.1. Basic Demographic and Clinicopathological Characteristics of Patients. To reduce the impact of the possible biases on the conclusion of this study to the minimum, the cases with similar demographic and clinicopathological characteristics were collected. The records of 120 patients with HBVrelated HCC treated in our department were retrospectively analysed. 40 cases were assigned into each group. Among them, 40 patients received radical hepatectomy alone (control group), 40 received radical hepatectomy and postoperative TACE (TACE group), and the remaining 40 received combination therapy of postoperative TACE and antiviral therapy after radical hepatectomy (combined group). The basic demographic and clinicopathological characteristics of patients before and after operation are shown in Tables 1 and 2. Among the three groups, no statistical significance $(P>0.05)$ was found for postoperative tumour characteristics and preoperative characteristics, which include the following: gender; age; counts of WBC, RBC, Hb, and PLT; and levels of ALT, AST, ALP, tumour markers (AFP, CA199, and CEA), and hepatitis B virus markers. Among the demographic characteristics, the patient number of ascites in the control group was different with that in the TACE group and combined group $(P=0.001, P=0.000$, resp.), and the total serum protein was different between the TACE group and combined group $(P=0.029)$, but the liver functions assessed by Child-Pugh class (consisting of ascites, hepatic encephalopathy, INR, total bilirubin, and total serum protein) showed no statistical significance $(P>0.05)$ (Supplementary Table 1). Median follow-up period was $54.26 \pm 22.65$ months with a range of 17-110 months. 
TABLE 2: Postoperative demographic characteristics of patients in the three groups.

\begin{tabular}{|c|c|c|c|c|c|}
\hline \multicolumn{2}{|c|}{ Postoperative patient demographic } & \multirow{2}{*}{$\begin{array}{c}\text { Control group } \\
1(2.5 \%)\end{array}$} & \multirow{2}{*}{$\frac{\text { TACE group }}{1(2.5 \%)}$} & \multirow{2}{*}{$\frac{\text { Combined group }}{1(2.5 \%)}$} & \multirow[t]{2}{*}{$P$ value } \\
\hline \multirow{4}{*}{ Edmondson grade } & $\mathrm{I}$ & & & & \\
\hline & II & $16(40.0 \%)$ & $18(45.0 \%)$ & $20(50.0 \%)$ & \multirow{3}{*}{0.677} \\
\hline & III & $15(37.5 \%)$ & $14(35 \%)$ & $13(32.5 \%)$ & \\
\hline & IV & $8(20.0 \%)$ & $7(17.5 \%)$ & $6(15.0 \%)$ & \\
\hline \multirow{2}{*}{ AJCC tumour stage } & I & $36(90.0 \%)$ & $34(85.0 \%)$ & $37(92.5 \%)$ & \multirow{2}{*}{0.549} \\
\hline & II & $4(10.0 \%)$ & $6(15.0 \%)$ & $3(7.5 \%)$ & \\
\hline \multirow{2}{*}{ Tumour number } & 1 & $36(90.0 \%)$ & $35(87.5 \%)$ & $37(92.5 \%)$ & \multirow{2}{*}{0.759} \\
\hline & 2 & $4(10.0 \%)$ & $5(12.5 \%)$ & $3(7.5 \%)$ & \\
\hline Maximum tumour size $(\mathrm{cm})$ & & $4.31 \pm 0.48$ & $4.01 \pm 0.67$ & $3.84 \pm 0.68$ & 0.004 \\
\hline \multirow{2}{*}{ Fibrous capsule formation } & Yes & $4(10.0 \%)$ & $5(12.5 \%)$ & $6(15.0 \%)$ & \multirow{2}{*}{0.797} \\
\hline & No & $36(90.0 \%)$ & $35(87.5 \%)$ & $34(85.0 \%)$ & \\
\hline \multirow{2}{*}{ Tumour invasion in capsule } & Yes & $0(0.0 \%)$ & $4(10.0 \%)$ & $3(7.5 \%)$ & \multirow{2}{*}{0.141} \\
\hline & No & $40(100.0 \%)$ & $36(90.0 \%)$ & $37(92.5 \%)$ & \\
\hline \multirow{2}{*}{ Microvascular invasion } & Yes & $6(15.0 \%)$ & $8(20.0 \%)$ & $4(10.0 \%)$ & \multirow{2}{*}{0.459} \\
\hline & No & $34(85.0 \%)$ & $32(80.0 \%)$ & $36(90.0 \%)$ & \\
\hline \multirow{2}{*}{ Portal vein invasion } & Yes & $0(0.0 \%)$ & $0(0.0 \%)$ & $0(0.0 \%)$ & \multirow{2}{*}{1.000} \\
\hline & No & $40(100.0 \%)$ & $40(100.0 \%)$ & $40(100.0 \%)$ & \\
\hline \multirow{2}{*}{ Serosa invasion } & Yes & $0(0.0 \%)$ & $1(2.5 \%)$ & $0(0.0 \%)$ & \multirow{2}{*}{0.368} \\
\hline & No & $40(100.0 \%)$ & $39(97.5 \%)$ & $40(100.0 \%)$ & \\
\hline \multirow{2}{*}{ Satellite nodule } & Yes & $4(10.0 \%)$ & $7(17.5 \%)$ & $4(10.0 \%)$ & \multirow{2}{*}{0.507} \\
\hline & No & $36(90.0 \%)$ & $33(82.5 \%)$ & $36(90.0 \%)$ & \\
\hline \multirow{2}{*}{ Surgical margin invasion } & Yes & $0(0.0 \%)$ & $0(0.0 \%)$ & $0(0.0 \%)$ & \multirow{2}{*}{1.000} \\
\hline & No & $40(100.0 \%)$ & $40(100.0 \%)$ & $40(100.0 \%)$ & \\
\hline Safety margin $(\mathrm{cm})$ & & $2.60 \pm 0.81$ & $2.85 \pm 1.05$ & $2.68 \pm 1.02$ & 0.497 \\
\hline \multirow{2}{*}{ p53 } & Positive & $16(40.0 \%)$ & $19(47.5 \%)$ & $20(50.0 \%)$ & \multirow{2}{*}{0.649} \\
\hline & Negative & $24(60.0 \%)$ & $21(52.5 \%)$ & $20(50.0 \%)$ & \\
\hline
\end{tabular}

3.2. Recurrence Rate of Patients in Different Groups. Recurrence after radical surgery was observed for 39 (97.5\%) patients in the TACE group, $32(80 \%)$ in the combined group, and 40 (100\%) in the control group with median time of 33, 43, and 16.5 months, respectively. Most recurrence cases were intrahepatic, such as in $27(67.5 \%)$ patients in the TACE group, $27(67.5 \%)$ in the combined group, and $23(57.5 \%)$ in the control group. Extrahepatic recurrence, mostly lung metastasis, was confirmed in $6(15.0 \%)$ patients in the TACE group, $1(2.5 \%)$ in the combined group, and $12(30.0 \%)$ in the control group.

3.3. DFS of Patients in Different Groups. Figure 1 shows the DFS rates of different groups and reveals that postoperative TACE with or without antiviral therapy significantly prolonged the DFS rate compared with radical hepatectomy alone $(P=0.000)$. Compared with the above two treatments, TACE combined with antiviral therapy significantly extended the DFS of patients compared with TACE alone $(P=0.008)$. Therefore, postoperative TACE may be beneficial for the DFS of patients, and its combination with antiviral therapy could further increase this effect.
3.4. OS of Patients in Different Groups. Death due to HCC progression or other tumour complications occurred in 27 (67.5\%) patients in the TACE group, 13 (32.5\%) in the combined group, and all $40(100 \%)$ patients in the control group with median OS rates of $64.50,63.50$, and 31 months, respectively. The OS rates of the three groups are presented in Figure 2. The combined group had significantly prolonged OS rate compared with the control group $(P=0.000)$. In addition, the 5-year OS rate of the combined group was significantly extended than that of the TACE and control groups $(67.5 \%$ versus $55 \%$ and $2.5 \%$; $P=0.032)$. Therefore, postoperative TACE and antiviral therapy may significantly prolong the OS rate of patients.

\section{Discussion}

HCC is the fifth most common malignancy in the world and causes approximately half a million deaths annually [16]. Surgical resection is the main treatment option for HCC. However, the high postoperative recurrence is the main obstacle for long-term survival. Various adjuvant treatments were developed with the hope of reducing recurrence rate and improving the OS of patients with HCC. 


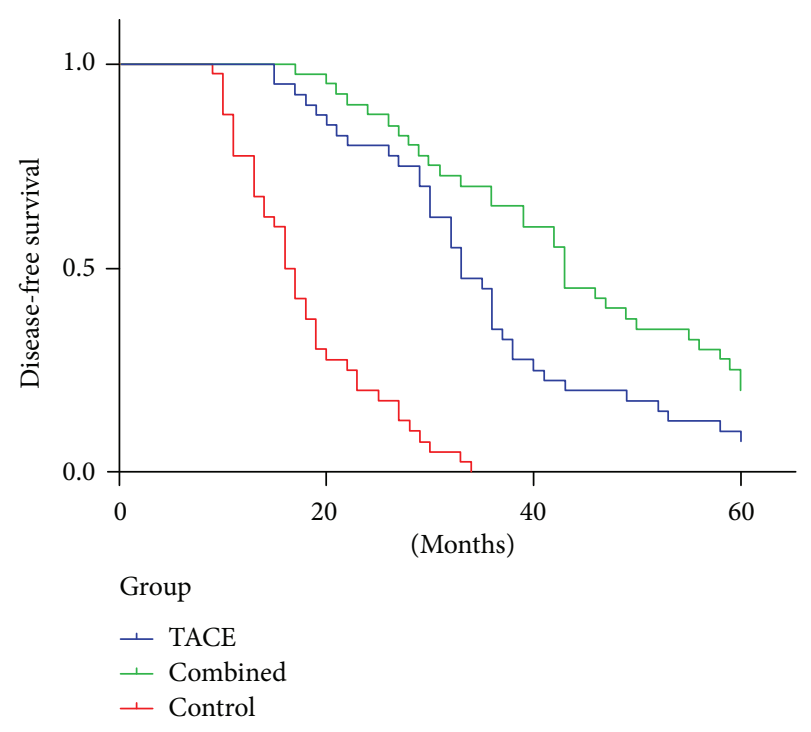

FIGURE 1: DFS of patients in different groups.

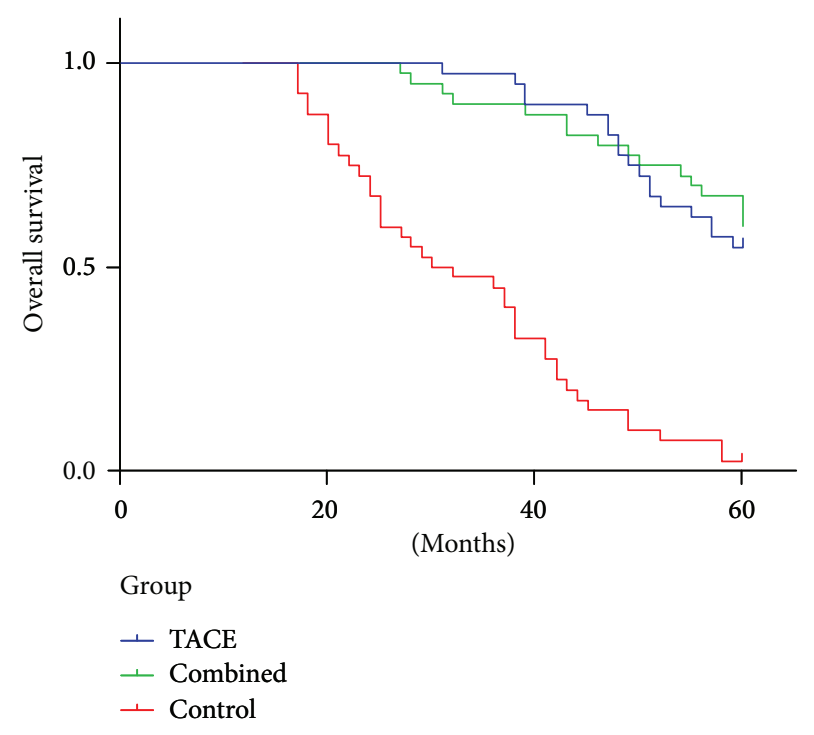

Figure 2: OS of patients in different groups.

TACE has definite therapeutic effects for patients with inoperable HCC [17]. Compared with systemically administered drugs, TACE increases the concentration of local chemotherapy drug, resulting in good antitumour response and low incidence of systemic toxicity-related side effects [18]. However, the efficacy of postoperative TACE for patients with HCC treated with curative hepatectomy remains controversial. Some studies showed unsatisfactory results of this adjuvant treatment $[7,8]$. A systematic review published in 2009 stated that no evidence suggests the efficacy of neoadjuvant/adjuvant protocols for the surgical resection of HCC [19]. The benefits of this treatment for postoperative patients require further studies. In addition, TACE might reactivate HBV replication after hepatectomy hypothetically due to its immunosuppression and cytotoxicity effects [20]. A related study also showed that the recurrence or metastasis rate dose dependently increases with the level of baseline HBV DNA; the range is from $22 \%$ for HBV DNA level of less than $3 \log _{10}$ copies $/ \mathrm{ml}$ to $80 \%$ for HBV DNA level of $5 \log _{10}$ copies/ml or higher [21]. Therefore, effectively controlling HBV replication using antiviral therapy may lower the risk of recurrence after liver resection for patients with HCC [22]. The recently developed oral anti-HBV nucleoside analogs show promise with their ability to naturally suppress hepatitis. Antiviral therapy with nucleoside analogs effectively reduces $\mathrm{HBV}$-induced liver damage, improves the liver function, promotes hepatocyte regeneration, and increases the volume of residual liver after hepatectomy [12, 23, 24]. However, limited credible evidence was found concerning the efficacy of combined postoperative TACE and antiviral therapy on HCC prognosis after radical tumour resection. Herein, we evaluated our medical data to explore the effect of postoperative TACE and antiviral therapy on the prognosis of HCC patients after radical hepatectomy.

Some authors suggested that adjuvant TACE is effective only for patients at high risk of recurrence due to multiple nodules and with $>5 \mathrm{~cm}$ tumour size and vascular invasion and/or narrow resection margin [25-27]. In our current study, few patients have satellite nodules $(4 / 40$, $7 / 40$, and $4 / 40$ ), the maximum tumour size was $>5 \mathrm{~cm}$ $(4.31 \pm 0.48 \mathrm{~cm}, 4.01 \pm 0.67 \mathrm{~cm}$, and $3.84 \pm 0.68 \mathrm{~cm})$, the resection margin exceeded $2.5 \mathrm{~cm}(2.60 \pm 0.81 \mathrm{~cm}, 2.85 \pm 1.05 \mathrm{~cm}$, and $2.68 \pm 1.02 \mathrm{~cm}$ ), and no patient had vascular invasion. To the best of our knowledge, a consensus is not yet established with regard to several aspects of postoperative TACE [28]. Most HCC recurrent tumours appeared 2 years after surgery; hence, many scholars believe that adjuvant TACE after surgery should be conducted as soon as possible. Their recommended schedule is 1 month postoperation. In our study, we performed TACE 2 months after the surgery when the patients had recovered from the operation. In addition, the frequency of postoperative TACE administration remains controversial. Some scholars believe that if patients with a liver function can tolerate it, then TACE should be repeated throughout the recurrence peak period. However, other scholars believe that repeatedly performing TACE may increase hepatic dysfunction and a single TACE treatment is superior to the repeated therapy [29]. In our current study, we repeated TACE 2 months after surgery depending on the patient's response to the first one. Finally, no consensus was established for the suitable chemotherapeutic agents, dosage and rate of injection [28]. Based on our experience, we selected 5-FU, DDP, and EPI as the treatment protocol. We also mixed the chemotherapeutic drugs with lipiodol to help increase their viscosity and X-ray visibility, thereby prolonging the chemotherapy-tumour interaction.

Our study showed that patients treated with TACE had a low recurrence rate. Recurrences occur because of preexisting microscopic tumour foci that are undetected due to imaging modalities or disseminated malignant cells during surgical manipulation. Thus, postoperative adjuvant TACE aims at eliminating the shed tumour cells that are potentially released through surgical manipulation and to destroy small intrahepatic metastases that may not be 
preoperatively detected [6]. Our results were consistent with the previous studies stating that TACE can effectively reduce the postoperative recurrence rate. In addition, most of the recurrence cases in our study were localised intrahepatically but not extrahepatically after the postoperative TACE. These cases have high probability to be considered eligible for reoperation. Considering that high HBV load and mutants promote HCC metastasis and growth [10] whereas hepatitis $\mathrm{B}$ virus $\mathrm{X}$ protein promotes the invasive ability and metastatic potential of HCCs, the combined treatment of TACE and antiviral therapy can further reduce the possibility of recurrence via inhibiting these viral factors.

In addition to reducing the risk of recurrence, postoperative TACE with or without antiviral treatment prolonged the DFS and OS rates compared with the radical hepatectomy alone $(P=0.000)$. TACE with antiviral therapy significantly improved the DFS and OS rates relative to postoperative TACE alone. We therefore suggest that the combination of postoperative TACE and antiviral therapy may be more effective than TACE alone in improving HCC prognosis. This result is consistent with a previous study from China, which reported significantly high OS and DFS rates for patients treated with combined TACE and lamivudine [14]. However, another study showed that lamivudine cannot improve the DFS rate [24]. Theoretically, due to the immunosuppression and cytotoxicity of regional chemotherapy, HBV reactivation and replication may happen during the perioperative period, especially for patients who do not receive antiviral therapy. Administering lamivudine to HCC patients after radical hepatectomy may reduce the levels of HBV DNA in the circulation and the risk of liver failure, significantly improve the liver function, and increase the possibility that radical surgery can be repeated in the event of recurrence [30]. According to the literature review of Zhong et al. [31], antivirus therapy with nucleoside analogs should be recommended for the following: (i) patients who are in the decompensation stage of cirrhosis or those who have ALT levels that are twice higher than the upper normal limit, (ii) patients with compensated cirrhosis and serum concentrations of $\mathrm{HBV}$ DNA $\geq 10^{4}$ copies/ml (if HbeAg positive) or $\geq 10^{3}$ copies $/ \mathrm{mL}$ (if HbeAg negative) regardless of the ALT level, and (iii) patients whose liver biochemistry findings are within the reference ranges when the serum concentrations of HBV DNA is $>10^{5}$ copies $/ \mathrm{ml}$. In our study, we included patients with the above-mentioned criteria. Nucleoside analogs naturally prevent the deterioration of liver function and thus enhance the tolerance to subsequent therapy such as TACE. This phenomenon may be the reason why patients with combined therapy had good DFS rate. DFS is proposed as an alternative for OS. TACE combined with lamivudine prevents the recurrence of disease and simultaneously improves the DFS and OS rates.

Our findings suggest that TACE is an appropriate therapy for HCC patients after radical hepatectomy. When combined with antiviral therapy, this treatment may further prolong the time of recurrence, leading to good DFS and OS rates. However, due to the nonrandomisation and small sample size of our study, further randomised studies with larger numbers of patients and longer follow-up periods are necessary to clarify whether TACE combined with antiviral therapy can improve the prognosis of patients with HCC after radical hepatectomy.

\section{Conflicts of Interest}

The authors declare that they have no conflicts of interest.

\section{Authors' Contributions}

Yin Zhu and Pei-Jing Cui contributed equally to this work.

\section{Acknowledgments}

This work was supported by a grant from the National Natural Science Foundation of China (81501086).

\section{Supplementary Materials}

Supplementary materials for supplementary Table 1 : patients' demographic data were retrospectively collected from the database. Demographic data were described using medians or frequencies. One-way ANOVA or chi-square test was used for the comparison of different groups. All statistical tests reached statistical significance at $P<0.05$. (Supplementary Materials)

\section{References}

[1] S. Chacko and S. Samanta, "Hepatocellular carcinoma: a lifethreatening disease," Biomedicine \& Pharmacotherapy, vol. 84, pp. 1679-1688, 2016.

[2] H. E. Blum, "Hepatocellular carcinoma: therapy and prevention," World Journal of Gastroenterology, vol. 11, no. 47, pp. 7391-7400, 2005.

[3] G. Fattovich, T. Stroffolini, I. Zagni, and F. Donato, "Hepatocellular carcinoma in cirrhosis: incidence and risk factors," Gastroenterology, vol. 127, no. 5, Supplement 1, pp. S35-S50, 2004.

[4] H. Imamura, Y. Matsuyama, E. Tanaka et al., "Risk factors contributing to early and late phase intrahepatic recurrence of hepatocellular carcinoma after hepatectomy," Journal of Hepatology, vol. 38, no. 2, pp. 200-207, 2003.

[5] H. M. Goldstein, S. Wallace, J. H. Anderson, R. L. Bree, and C. Gianturco, "Transcatheter occlusion of abdominal tumors," Radiology, vol. 120, no. 3, pp. 539-545, 1976.

[6] J. H. Zhong and L. Q. Li, "Postoperative adjuvant transarterial chemoembolization for participants with hepatocellular carcinoma: a meta-analysis," Hepatology Research, vol. 40, no. 10, pp. 943-953, 2010.

[7] J. W. Peng, G. N. Lin, J. J. Xiao, and X.-M. Jiang, "Hepatitis B virus reactivation in hepatocellular carcinoma patients undergoing transcatheter arterial chemoembolization therapy," Asia-Pacific Journal of Clinical Oncology, vol. 8, no. 4, pp. 356-361, 2012.

[8] J. W. Jang, J. Y. Choi, S. H. Bae et al., "Transarterial chemolipiodolization can reactivate hepatitis B virus replication in patients with hepatocellular carcinoma," Journal of Hepatology, vol. 41, no. 3, pp. 427-435, 2004. 
[9] C. I. Lin, Z. Y. Lin, M. Y. Hsieh, C. F. Huang, S. H. Chen, and W. L. Chuang, "Potential risk factors for the reactivation of the replication of hepatitis $\mathrm{B}$ and $\mathrm{C}$ viruses after transcatheter arterial chemoembolization of hepatocellular carcinoma," Kaohsiung Journal of Medical Sciences, vol. 27, no. 12, pp. 554-559, 2011.

[10] I. F. Hung, R. T. Poon, C. L. Lai, J. Fung, S.-T. Fan, and M.-F. Yuen, "Recurrence of hepatitis B-related hepatocellular carcinoma is associated with high viral load at the time of resection," The American Journal of Gastroenterology, vol. 103, no. 7, pp. 1663-1673, 2008.

[11] M. F. Yuen and C. L. Lai, "Treatment of chronic hepatitis B: evolution over two decades," Journal of Gastroenterology and Hepatology, vol. 26, no. s1, pp. 138-143, 2011.

[12] T. Kuzuya, Y. Katano, T. Kumada et al., "Efficacy of antiviral therapy with lamivudine after initial treatment for hepatitis B virus-related hepatocellular carcinoma," Journal of Gastroenterology and Hepatology, vol. 22, no. 11, pp. 1929-1935, 2007.

[13] Q. Yan, J. Ni, G. L. Zhang et al., "Efficacy of post-operative antiviral combined transcatheter arterial chemoembolization therapy in prevention of hepatitis B-related hepatocellular carcinoma recurrence," Chinese Medical Journal, vol. 126, no. 5, pp. 855-859, 2013.

[14] S. L. Zhu, J. H. Zhong, Y. Ke et al., "Comparative efficacy of postoperative transarterial chemoembolization with or without antiviral therapy for hepatitis B virus-related hepatocellular carcinoma," Tumour Biology, vol. 36, no. 8, pp. 6277-6284, 2015.

[15] J. Q. Li, Y. Q. Zhang, W. Z. Zhang, Y. F. Yuan, and G. H. Li, "Randomized study of chemoembolization as an adjuvant therapy for primary liver carcinoma after hepatectomy," Journal of Cancer Research and Clinical Oncology, vol. 121, no. 6, pp. 364-366, 1995.

[16] Y. M. Zhou, J. M. Yang, B. Li et al., "Risk factors for early recurrence of small hepatocellular carcinoma after curative resection," Hepatobiliary \& Pancreatic Diseases International, vol. 9, no. 1, pp. 33-37, 2010.

[17] K. W. Li, X. Li, T. F. Wen, and W. S. Lu, "The effect of postoperative TACE on prognosis of HCC: an update," HepatoGastroenterology, vol. 60, no. 122, pp. 248-251, 2013.

[18] D. Ippolito, P. A. Bonaffini, L. Ratti et al., "Hepatocellular carcinoma treated with transarterial chemoembolization: dynamic perfusion-CT in the assessment of residual tumor," World Journal of Gastroenterology, vol. 16, no. 47, pp. 59936000, 2010.

[19] M. Samuel, P. K. Chow, E. Chan Shih-Yen, D. Machin, and K.-C. Soo, "Neoadjuvant and adjuvant therapy for surgical resection of hepatocellular carcinoma," Cochrane Database of Systematic Reviews, no. 1, 2009.

[20] A. S. F. Lok, R. H. S. Liang, E. K. W. Chiu, K.-L. Wong, T.-K. Chan, and D. Todd, "Reactivation of hepatitis B virus replication in patients receiving cytotoxic therapy. Report of a prospective study," Gastroenterology, vol. 100, no. 1, pp. 182-188, 1991.

[21] Y. Huang, Z. Wang, S. An et al., "Role of hepatitis B virus genotypes and quantitative HBV DNA in metastasis and recurrence of hepatocellular carcinoma," Journal of Medical Virology, vol. 80, no. 4, pp. 591-597, 2008.

[22] C. Shuqun, W. Mengchao, C. Han et al., "Antiviral therapy using lamivudine and thymosin alpha1 for hepatocellular carcinoma coexisting with chronic hepatitis B infection," HepatoGastroenterology, vol. 53, no. 68, pp. 249-252, 2006.
[23] C. Y. Piao, S. Fujioka, Y. Iwasaki et al., "Lamivudine treatment in patients with HBV-related hepatocellular carcinoma-using an untreated, matched control cohort," Acta Medica Okayama, vol. 59, no. 5, pp. 217-224, 2005.

[24] N. Li, E. C. Lai, J. Shi et al., "A comparative study of antiviral therapy after resection of hepatocellular carcinoma in the immune-active phase of hepatitis B virus infection," Annals of Surgical Oncology, vol. 17, no. 1, pp. 179-185, 2010.

[25] J. H. Zhong, L. Ma, and L. Q. Li, "Postoperative therapy options for hepatocellular carcinoma," Scandinavian Journal of Gastroenterology, vol. 49, no. 6, pp. 649-661, 2014.

[26] Z. G. Ren, Z. Y. Lin, J. L. Xia et al., "Postoperative adjuvant arterial chemoembolization improves survival of hepatocellular carcinoma patients with risk factors for residual tumor: a retrospective control study," World Journal of Gastroenterology, vol. 10, no. 19, pp. 2791-2794, 2004.

[27] K. Tanaka, H. Shimada, S. Togo et al., "Use of transcatheter arterial infusion of anticancer agents with lipiodol to prevent recurrence of hepatocellular carcinoma after hepatic resection," Hepato-Gastroenterology, vol. 46, no. 26, pp. 10831088, 1999.

[28] W. Y. Lau, E. C. Lai, and S. H. Lau, "The current role of neoadjuvant/adjuvant/chemoprevention therapy in partial hepatectomy for hepatocellular carcinoma: a systematic review," Hepatobiliary \& Pancreatic Diseases International, vol. 8, no. 2, pp. 124-133, 2009.

[29] T. Xi, Z. L. Yan, K. Wang et al., "Role of post-operative transcatheter arterial chemoembolization in hepatocellular carcinoma with different pathological characteristics," Chinese Journal of Surgery, vol. 45, no. 9, pp. 587-590, 2007.

[30] X. M. Lao, G. Luo, L. T. Ye et al., "Effects of antiviral therapy on hepatitis B virus reactivation and liver function after resection or chemoembolization for hepatocellular carcinoma," Liver International, vol. 33, no. 4, pp. 595-604, 2013.

[31] J. H. Zhong, Q. L. Zhong, L. Q. Li, and H. Li, “Adjuvant and chemopreventive therapies for resectable hepatocellular carcinoma: a literature review," Tumour Biology, vol. 35, no. 10, pp. 9459-9468, 2014. 


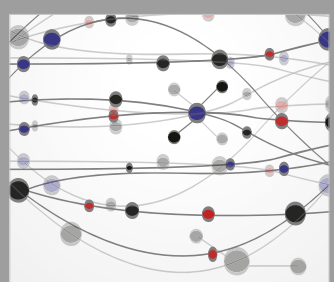

The Scientific World Journal
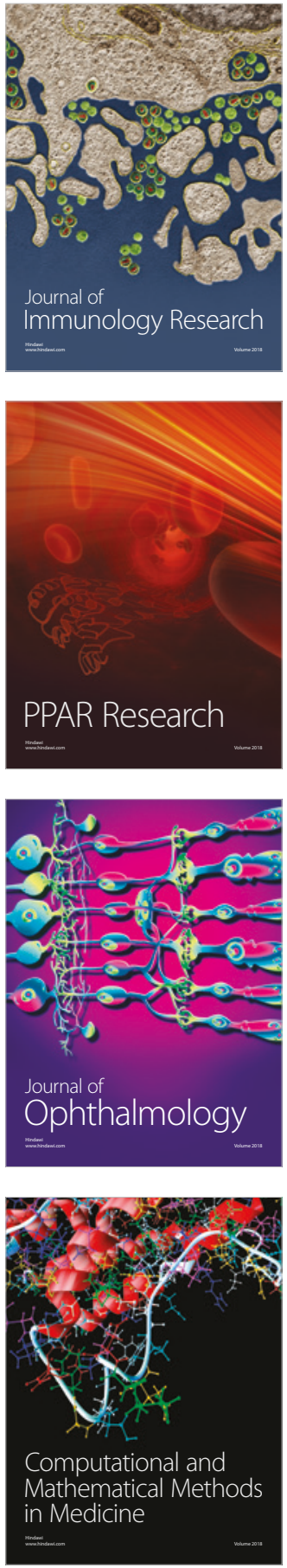

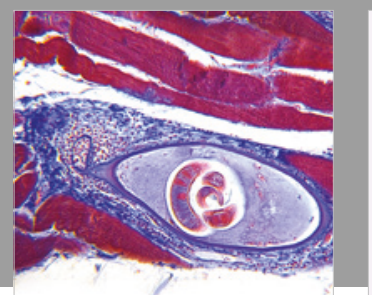

Gastroenterology Research and Practice

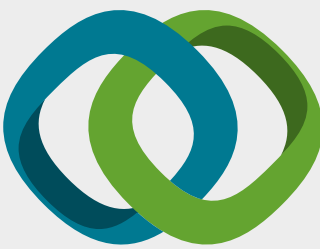

\section{Hindawi}

Submit your manuscripts at

www.hindawi.com
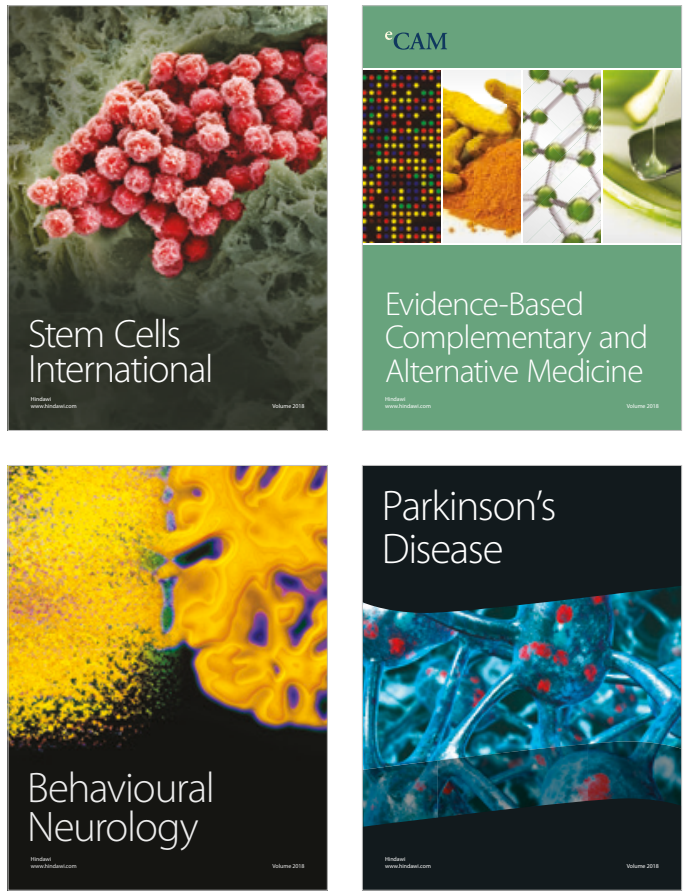

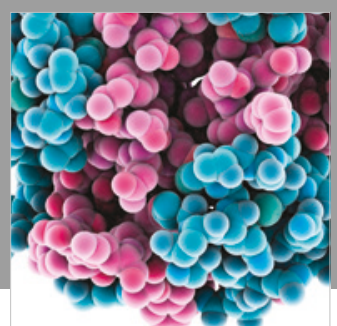

ournal of

Diabetes Research

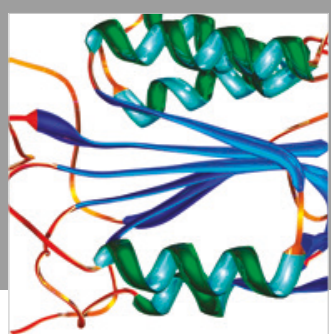

Disease Markers
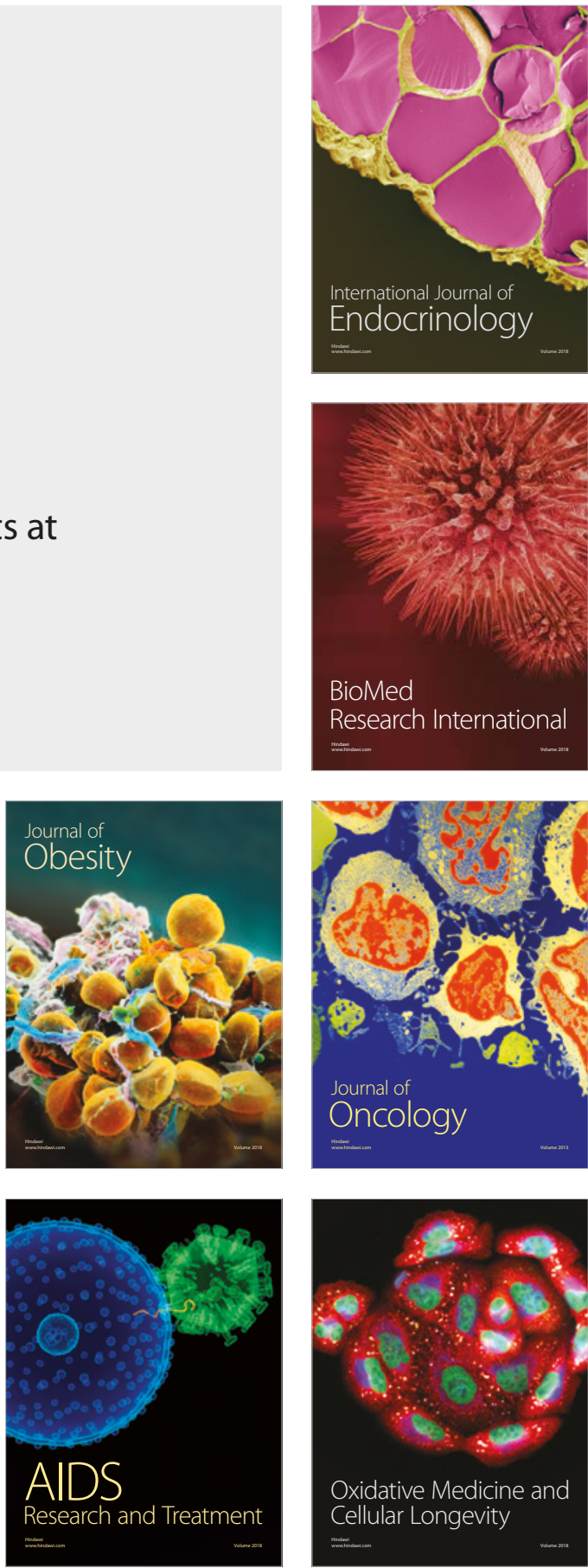